\section{Electrochemical performance of nanodiamond and multiwalled carbon nanotubes/nanodiamond composites in electrical double layer capacitors}

\section{E.C. Almeida $^{1}$, L.I. Medeiros ${ }^{1}$, M.R. Baldan ${ }^{1}$, J.M. Rosolen $^{2}$, N.G. Ferreira ${ }^{1}$}

${ }^{1}$ Instituto Nacional de Pesquisas Espaciais- INPE, São José dos Campos, 12.245-970, Brazil;

${ }^{2}$ Departamento de Química, FFCLRP, Universidade de São Paulo, 14040-901 Ribeirão Preto, Brazil

The purpose of this work is to examine the performance of the nanodiamond and multiwalled nanotubes/nanodiamond composites growth on carbon fiber substrate as an electrochemical capacitor. The influence of the substrate carbonization temperature on nanodiamond surface morphology and the electrochemical response were examined. The electrochemical characterization of the electrodes was determined by cyclic voltammetry (CV), charge-discharge and impedance analysis. Carbon fibers were produced from a polyacrylonitrile at three carbonization temperature: 1000, 1500 and $2000{ }^{\circ} \mathrm{C}$. Nanodiamond films were grown by the Hot Filaments Chemical Vapor Deposition technique with a mixture of $1 \mathrm{vol} \% \mathrm{CH}_{4}, 90$ vol\% $\mathrm{Ar}$ and 9 vol\% de $\mathrm{H}_{2}$. The nanodiamond films grown on carbon fibers substrates showed a rounded appearance, ball-like or cauliflower-like morphology as may be observed in Figure 1.

The multiwalled nanotubes were deposited on nanodiamond films by electrophoretic deposition. The nanotube materials were dispersed in water by ultrasonication to obtain dispersions of $0.05 \mathrm{mg} / \mathrm{mL}$. The nanodiamond films were immersed in the nanotube dispersion, and a positive potential of $10 \mathrm{~V} / \mathrm{cm}$ was applied. Figure 2 shows the multiwalled nanotubes/nanodiamond composites on carbon fiber substrate, a homogeneous deposition of multiwalled carbon nanotubes on the surface of the nanodiamond were obtained with success.

Electrochemical response of electrodes was analyzed in aqueous and organic electrolyte. The electrolyte aqueous was $0.5 \mathrm{M}$ of $\mathrm{H}_{2} \mathrm{SO}_{4}$ solutions and platinum and $\mathrm{Ag} / \mathrm{AgCl}$ was used as counter and reference electrodes, respectively. The organic electrolyte was mixture 1:1 $\mathrm{PC} / \mathrm{EC}$ in $1 \mathrm{M} \mathrm{LiClO}_{4}$ and $\mathrm{Li}$ metallic was used as counter and reference electrode.

Figure 3 shows the $\mathrm{CV}$ curves measured from the nanodiamond/carbon fibers, in $0.5 \mathrm{M} \mathrm{H}_{2} \mathrm{SO}_{4}$ at two different voltage scan rates. The capacitive behavior of an ideal electrode is expressed in the form of a rectangular shape of the CV. In Figure 3, the nanodiamond/carbon fiber shows the best rectangular shape capacitor. The capacitive behavior is associated to its surface area increase that influenced the active area accessible to the electrolyte.

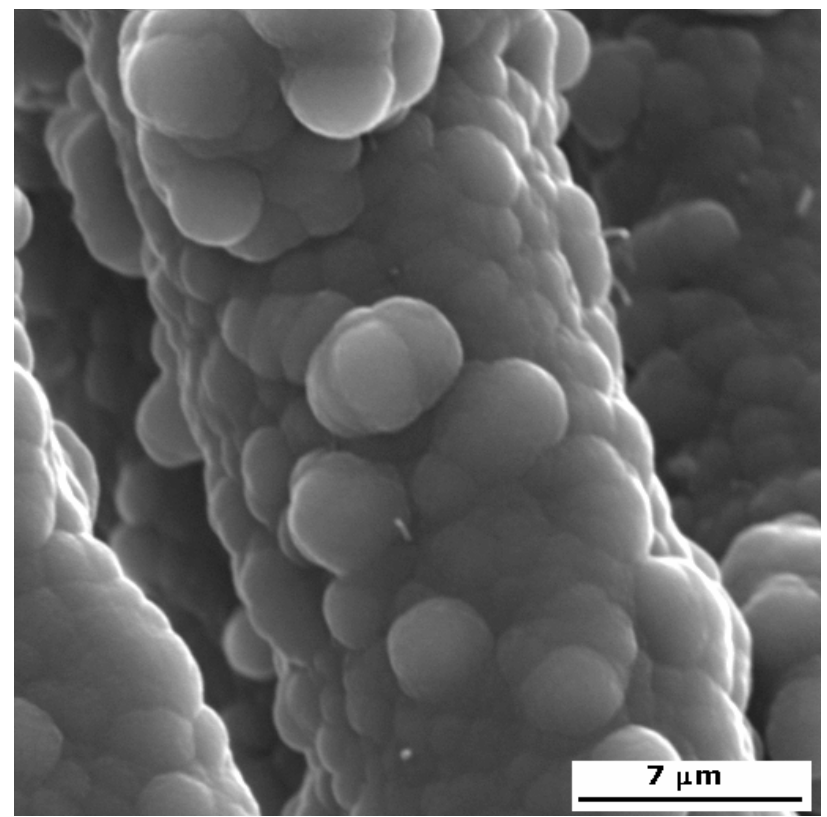

Figure 1. SEM image of nanodiamond growth on carbon fiber substrate.

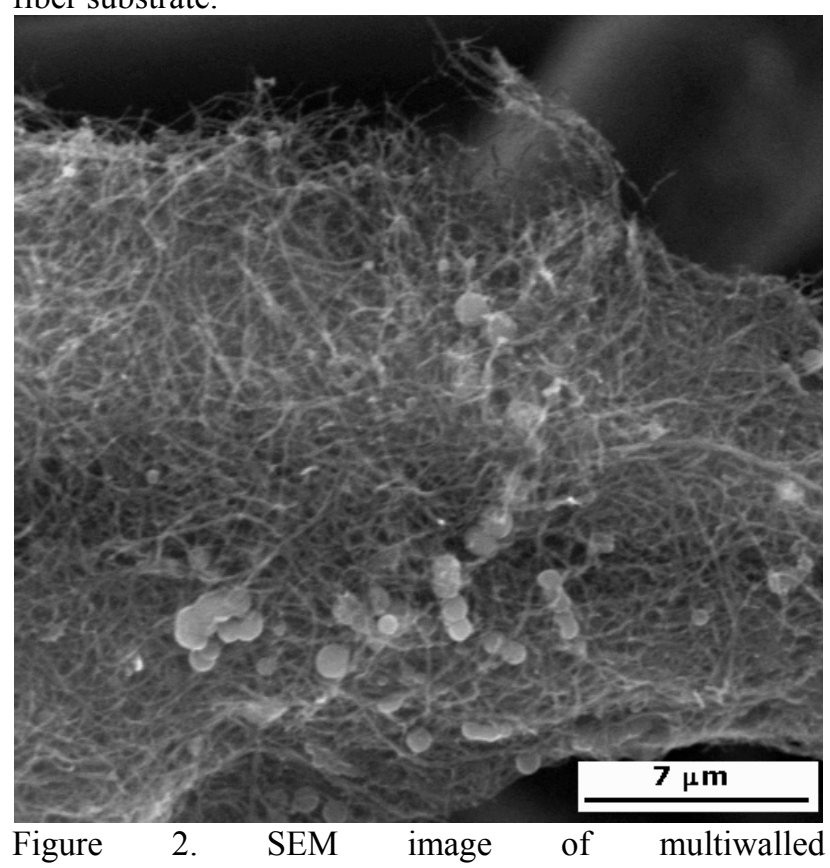

nanotubes/nanodiamond composites growth on carbon fiber substrate

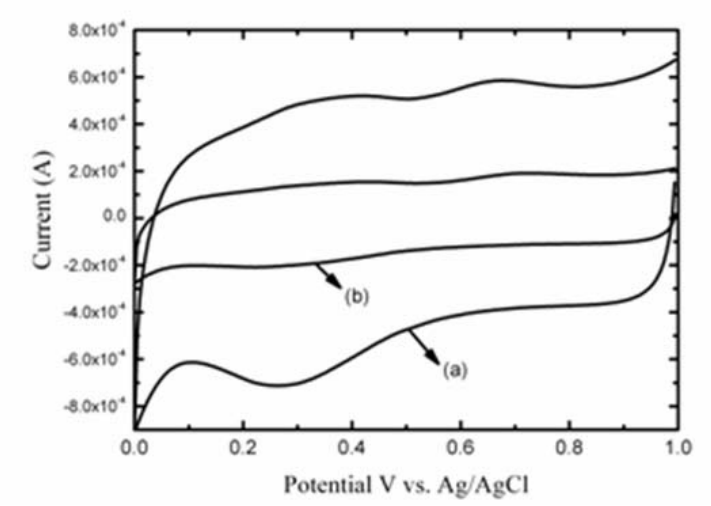

Figure 3. Cyclic voltammetric behavior at $100 \mathrm{mV} / \mathrm{s}$ (a) and $10 \mathrm{mV} / \mathrm{s}$ (b) in $0.5 \mathrm{M}$ of $\mathrm{H}_{2} \mathrm{SO}_{4}$ solutions of nanodiamond grown on carbon fibers. 